\title{
An Optical Design of Off-axis Four-mimor-anastigmatic Telescope for Remote Sensing
}

\author{
Xing long $\mathrm{Li}^{1}$, Min $\mathrm{Xu}^{1 *}$, Xian dong $\mathrm{Ren}^{2}$, and Yun tian $\mathrm{Pei}^{3}$ \\ ${ }^{1}$ Department of Optical Science and Optical Engineering, Fudan University, \\ 220 Handan Road, Shanghai 2000433, China \\ ${ }^{2}$ School of Information Engineering of Jining Medical University, \\ 669 Xueyuan Road, Rizhao 276826, China \\ ${ }^{3}$ Shanghai Institute of Technical Physics of the Chinese Academy of Sciences, \\ 500 Yutian Road, Shanghai 2000083, China
}

(Received May 31, 2012 : revised July 5, 2012 : accepted July 6, 2012)

\begin{abstract}
An off-axis four-mirror-anastigmatic telescope is presented here which is composed of two aspheric surfaces and two spherical surfaces. The entrance pupil diameter is $290 \mathrm{~mm}$ and the stop is located at the primary mirror. The effective focal length is $900 \mathrm{~mm}$. The strip field of view for the telescope is $15^{\circ} \times 0.2^{\circ}$ and if the telescope is launched into an orbit about $400 \mathrm{~km}$ altitude, the observed range width will be more than $105 \mathrm{~km}$ within a scene without any other auxiliary scanning instrument. The spectral range can be as wide as from visual wave band to infrared wave band in the mirror system. This telescope can be used for environmental monitoring with different detectors whose pixel is adapted to the optical resolution. In this paper, the spectral range is chosen as 3.0 -5.0 $\mu \mathrm{m}$, and center distance of the pixel is $30 \mu \mathrm{m}$. And the image quality is near the diffraction limit.
\end{abstract}

Keywords : Optical design, Mirror, Telescope, Space optics, Off-axis

OCIS codes : (080.2740) Geometric optical design; (080.4035) Mirror system design; (120.4570) Optical design of instruments

\section{INTRODUCTION}

Recently, due to the rapid development of remote sensing, reflective telescopes are widely used, since large dimension optical refractive material is not required in the telescopes; and there are no chromatic aberrations in the optical path. The field of view (FOV) is small in the two-mirror optical telescopes, for example, the FOV for the Ritchey-Chretien optical system which has largest FOV is only a few minutes [1]. Though the catadioptric telescope can give a much larger FOV as $2.3^{\circ}$ in STSAT3 [2], the refractive material for the telescope is also large especially when the telescope configuration is used as a high resolution remote sensing instrument. FOV is as wide as $10^{\circ}$ in the reflective Schmidt telescope, but it has a very large configuration $[3,4]$. The off-axis three-mirror-anastigmatic (TMA) telescope can give a wider FOV and a more compact configuration $[5,6]$, but the off-axis TMA telescope is difficult to achieve because of three aspheric mirrors, especially for off-axis TMA telescope with strip FOV wider than $10^{\circ}$.

With the development of space optical remote sensing, the wider spectral range and the wider FOV are pursued. Wider spectral range in the telescope can provide more information from the interested targets, wider FOV can provide wider range in the object space without any other auxiliary scanning instrument since the width is given by equation 1 .

$$
d=2 h \times \tan (F O V / 2)
$$

where $\mathrm{h}$ is the altitude of the telescope to earth. When $\mathrm{h}$ is not changed, the wider FOV, the larger $d$, then the function of the telescope will be improved. The geometric relationship about $\mathrm{d}, \mathrm{h}$ and FOV is shown in Fig. 1.

When the FOV is very big such as more than $10^{\circ}$, then the apertures for high resolution reflective telescope (long focal length and large entrance pupil) will be larger and the difficulties for aspheric mirror fabrication will be sharply

*Corresponding author: minx@fudan.edu.cn 


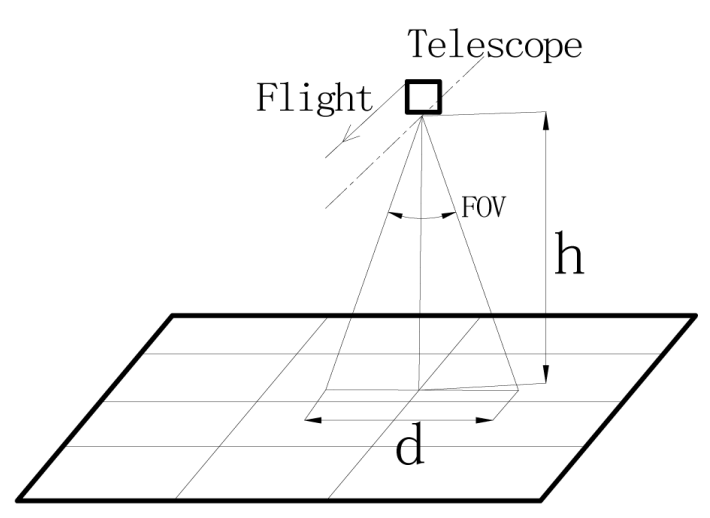

FIG. 1. Geometrical relationship about $\mathrm{d}$, h and FOV.

increased. In order to achieve a telescope with high resolution, wide FOV, and easy manufacturing, an off-axis four-mirroranastigmatic (FMA) telescope with $290 \mathrm{~mm}$ entrance pupil was designed which is composed of two aspheric mirrors and two spherical mirrors. And the stop is located at the primary mirror. The strip FOV for the telescope is $15^{\circ} \times$ $0.2^{\circ}$ and the effect focal length is $900 \mathrm{~mm}$.

\section{OPTICAL DESIGN OF OFF-AXIS FOUR-MIRROR-ANASTIGMATIC TELESCOPE}

\subsection{Optical Design Requirements}

There should be no obscuration in the optical path in order to improve the optical MTF (Modulate Transfer Function). Then the off-axis mode will be taken. In the telescope, the field of view is tilted about the $\mathrm{X}$ axis (which is perpendicular to the optical axis, and the Cartesian coordinates shown in Fig. 2), and the stop is located at the primary mirror.

In order to detect the environmental targets whose irradiance can pass through the atmosphere, the spectral range is chosen as $3.0-5.0 \mu \mathrm{m}$. In the optical designing process, the diffraction effect should be of concern since the detectors can be chose scientifically in order to collect more energy from instant FOV by every pixel. The radius of Airy disk can be deduced by equation 2 .

$$
r=\frac{1.22 \lambda}{D} f=1.22 \lambda F / \#
$$

Where $\lambda$ is the primary wavelength, $\mathrm{D}$ is the entrance pupil diameter, $f$ is the focal length of telescope, and $F / \#$ is the number of $\mathrm{f} / \mathrm{D}$. In this telescope, the primary wavelength $\lambda=4 \mu \mathrm{m}$, the $\mathrm{F} / \#=900 / 290=3.1$, so that the radius of the Airy disk $\mathrm{r}=15.145 \mu \mathrm{m}$, then the diameter of the Airy disk is $30.29 \mu \mathrm{m}$. And center distance of the pixel is $30 \mu \mathrm{m}$ in the detector, so that most of the energy within an instant FOV can be collected by each pixel. Then the instant FOV can be deduced by the equation 3 .

$$
\operatorname{InFOV}=\frac{b}{f}
$$

Where $\mathrm{b}$ is the center distance of the pixel, $\mathrm{f}$ is the focal length of the telescope. Then the angular resolution is 33.3 $\times 10^{-6}$ radian, the ground sample distance (GSD) is about $13.33 \mathrm{~m}$ on the earth's surface when the altitude is 400 $\mathrm{km}$. Since the FOV is $15^{\circ} \times 0.2^{\circ}$, then the focal plane of the detector is about $7850 \times 100$ pixels. When the altitude is $400 \mathrm{~km}$ and the push-broom mode is used, the area on the earth's surface is about $105 \mathrm{~km} \times 1.39 \mathrm{~km}$ within a scene.

\subsection{Optical Design Results}

In order to get wider FOV and decrease the manufacturing difficulty, we can increase a mirror to the TMA telescope to redistribute the power as $\Phi=\sum_{i=1}^{4} \Phi_{i}$, where $\Phi$ is the power of the FMA telescope, and $\Phi_{i}$ is the power of the $i^{\text {th }}$ mirror in the FMA telescope. The third aspheric mirror in the TMA telescope can be designed as two spherical mirrors in the FMA telescope.

In order to remove the obscuration in the telescope, two methods can be taken:

(1) Make the incident beam tilt and propagate to the telescope in the Cartesian coordinates;

(2) Make the entrance pupil be off-axis in the Cartesian coordinates.

The telescope is designed as off-axis FMA telescope, which is composed of two aspheric mirror and two spherical mirrors. In the telescope, the primary mirror (PM) is a symmetric concave aspheric mirror, the secondary mirror (SM) is a deviated (along the $\mathrm{Y}$ axis which is perpendicular to optical axis, and the Cartesian coordinates shown in Fig. 2) convex aspheric mirror, and the third mirror (TM) and the fourth mirror (FM) are all concave spherical mirrors. The entrance pupil is located at the PM. The optical configuration of the off-axis FMA telescope is shown as Fig. 2, the lens data is shown in Table 1 from ZEMAX

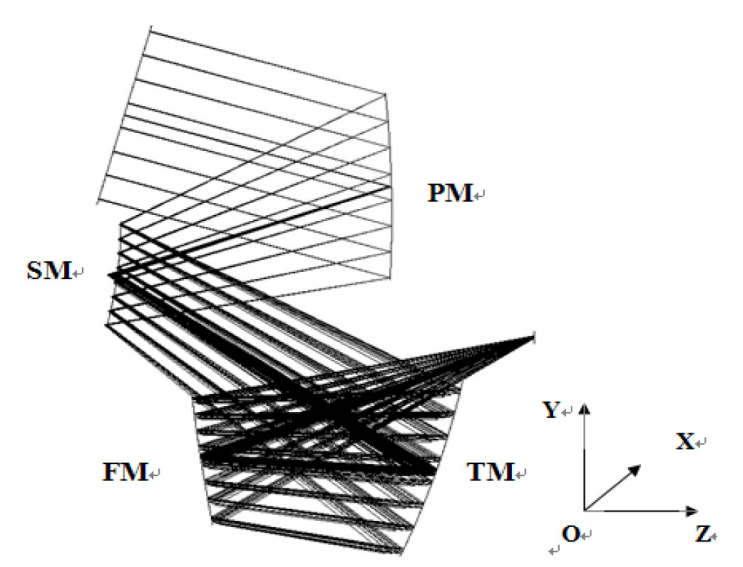

FIG. 2. Optical configuration of off-axis FMA telescope. 
An Optical Design of Off-axis Four-mirror-anastigmatic Telescope for Remote Sensing - Xing long Li et al. 245

TABLE 1. Lens data from ZEMAX (unit: thickness/distance in mm, angle in deg.)

\begin{tabular}{c|c|c|c|c|c}
\hline \hline Surface & Type & Radius & Thickness & Glass & Tilt X \\
\hline OBJ & Standard & Infinity & Infinity & Air & \\
\hline 1 & Standard & Infinity & 520 & & -14 \\
\hline 2 & Coordinate Break & & & Mirror & \\
\hline STO & Even Asphere & -2154.98 & -484.52 & Mirror & \\
\hline 4 & Even Asphere & -675.78 & 549.22 & Mirror & Mirror \\
\hline 5 & Standard & -1341.08 & -480.00 & Air & \\
\hline IMG & Standard & 2881.90 & 543.04 & & \\
\hline
\end{tabular}

TABLE 2. Parameters for PM

\begin{tabular}{c|c|c}
\hline \hline Conic $(\mathrm{k})$ & $4^{\text {th }}$ Order Term & $6^{\text {th }}$ Order Term \\
\hline-2.76 & $-6.1 \mathrm{E}-12$ & $-9.78 \mathrm{E}-18$ \\
\hline
\end{tabular}
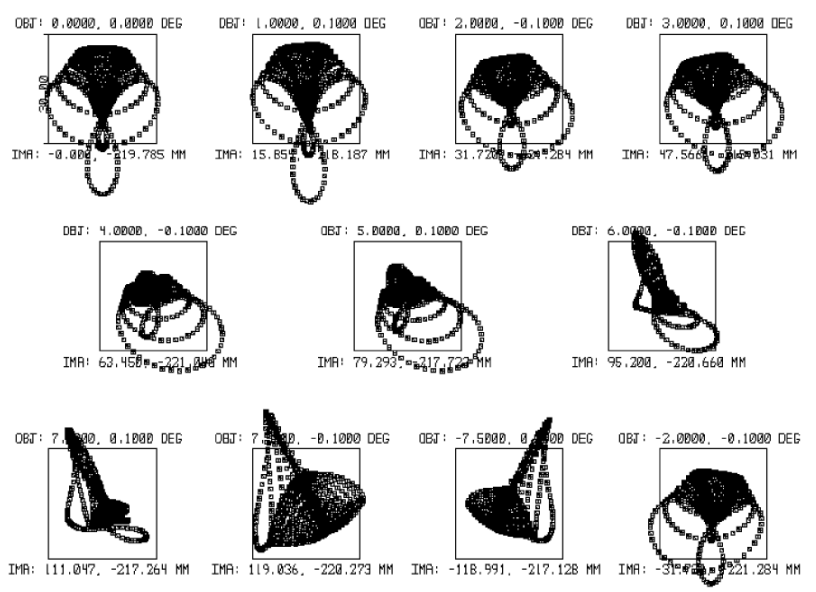

FIG. 3. Spot diagram for FMA telescope.

lens data format.

In this paper, the PM and SM are all even aspheric surfaces, and the surface sag is shown as equation 4.

$$
z=\frac{c r^{2}}{1+\sqrt{1-(1+k) c^{2} r^{2}}}+\sum_{i=1}^{8} \alpha_{1} r^{2 n}
$$

Where $\mathrm{c}$ is the curvature (the reciprocal of the radius), $\mathrm{r}$ is the radial coordinate in lens unit, $\mathrm{k}$ is the conic constant, and $\alpha_{1}$ is the coefficient.

The even aspheric surface parameters for PM and SM are shown in Table 2 and Table 3.

The image quality is evaluated by Spot Diagram, Optical MTF and Grid Distortion as shown in Fig 3, Fig 4 and Fig. 5. The maximum RMS radius of the spot diagram is $10.882 \mu \mathrm{m}$, when the pixel is $30 \mu \mathrm{m} \times 30 \mu \mathrm{m}$. At the Nyquist frequency (16.67 lines per millimeter), the minimum MTF
TABLE 3. Parameters for SM

\begin{tabular}{c|c|c}
\hline \hline Conic $(\mathrm{k})$ & $4^{\text {th }}$ Order Term & $6^{\text {th }}$ Order Term \\
\hline 1.17 & $5.04 \mathrm{E}-10$ & $1.42 \mathrm{E}-15$ \\
\hline
\end{tabular}

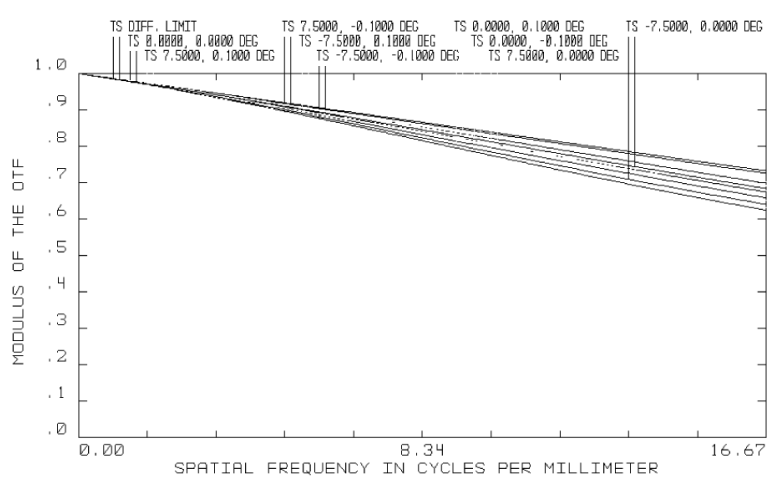

FIG. 4. MTF plot for FMA telescope.

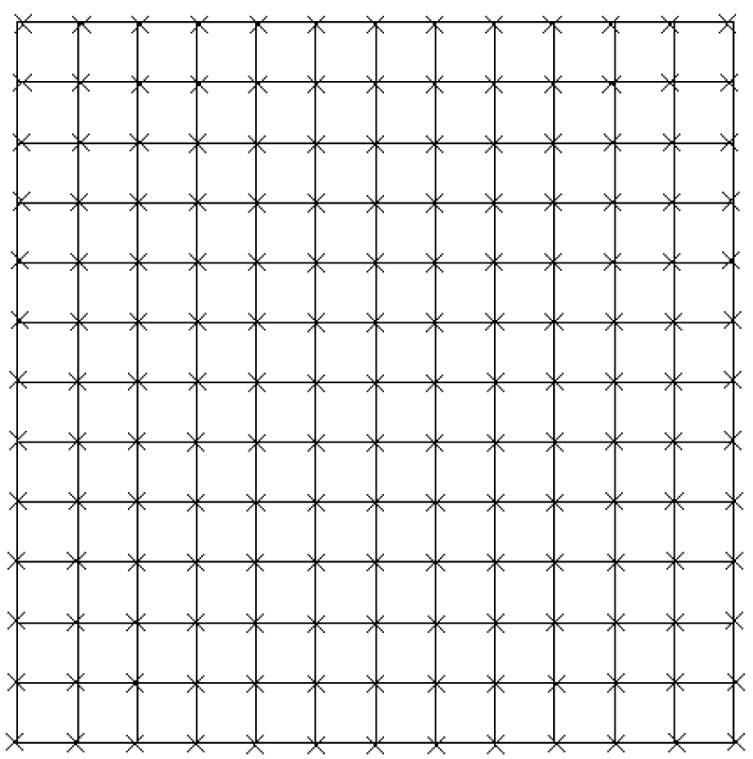

FIG. 5. Distortion for FMA telescope. 


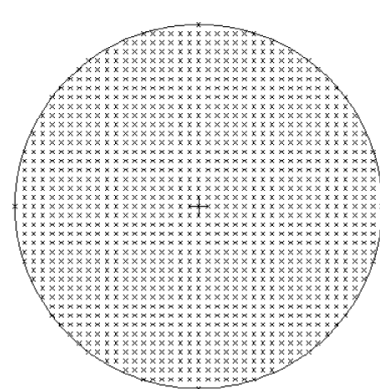

(a)

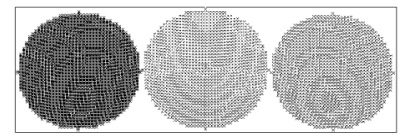

(c)

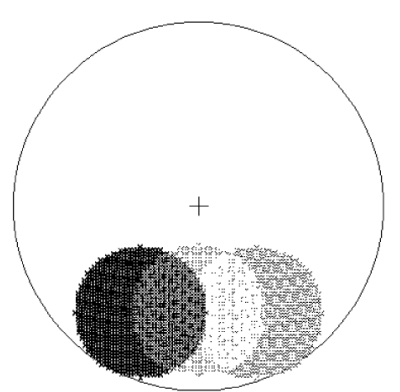

(b)

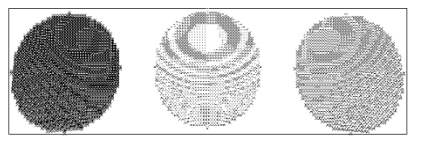

(d)
FIG. 6. Ray footprints on mirror surface.

TABLE 4. Optical tolerances for manufacturing

\begin{tabular}{l|l}
\hline \hline \multicolumn{1}{c|}{ Item } & Tolerances \\
\hline Surface of PM & \pm 5 fringe \\
\hline Surface of SM & \pm 4 fringe \\
\hline Surface of TM & \pm 5 fringe \\
\hline Surface of FM & \pm 5 fringe \\
\hline Spherical \& Astigmatism & \pm 2 fringe \\
\hline Thickness & $\pm 0.04 \mathrm{~mm}$ \\
\hline Decenter & $\pm 0.04 \mathrm{~mm}$ \\
\hline Tilt & $\pm 1.5 \mathrm{arcmin}$ \\
\hline
\end{tabular}

for the telescope is 0.623 . The maximum distortion is $-1.0237 \%$. The image quality is good enough for the remote sensing application.

The ray footprints of each mirror are shown as Fig. 6. The ray footprints for PM are shown in Fig. 6(a), it shows that the PM is a symmetric mirror so that the difficulty of fabrication can be decreased compared to the off-axis one. The ray footprints for SM are shown in Fig. 6(b), it shows that the convex mirror used in the optical path is deviated along the Y axis. Since TM and FM are spherical mirrors, they do not have a symmetric optical axis as the aspheric mirror, and then we do not care for their distances deviated along $\mathrm{Y}$ axis. The ray footprints for TM and FM are shown in Fig. 6(c) and Fig. 6(d).

\subsection{The Optical Tolerances for Manufacturing}

Just the manufacturing errors concerned, the optical tolerances are shown in Table 4, and the RMS radius for spot diagrams will be changed at most $2.1 \mu \mathrm{m}$ with Mont Carlo calculations in the ZEMAX program. From Table 4, we can conclude that the tolerances are loose.

\section{CONCLUSION}

An optical design of off-axis FMA (four-mirror-anastigmatic) telescope $(\mathrm{f} / 3.1)$ is presented in this paper. The telescope is composed of two aspheric mirrors and two spherical mirrors. The PM is a symmetric concave aspheric mirror, $\mathrm{SM}$ is a convex aspheric mirror, and TM and FM are all concave spherical mirrors. With spectral range 3.0 -5.0 $\mu \mathrm{m}$, the image quality is near diffraction limited. The strip FOV is $15^{\circ} \times 0.2^{\circ}$. From the altitude of $400 \mathrm{~km}$, the FMA telescope can take a scene of $105 \mathrm{~km} \times 1.39 \mathrm{~km}$ without any scanning instrument while the GSD is $13.33 \mathrm{~m}$.

\section{ACKNOWLEDGMENT}

This research was supported by the National Science and Technology Major Project of Ministry of Science and Technology of China under Grant NO. 2011zx02402, and the National High Technology R\&D Program of China (863 Program) under NO. 2012AA 040406.

\section{REFERENCES}

1. J. H. Pan, The Design, Manufacture and Test of the Aspheric Optical Surfaces (Science Press, Beijing, China, 1994), pp. 10-47.

2. J. H. Lee, T. S. Jang, H. S. Yang, and S. W. Rhee, "Optical design of a compact imaging spectrometer for STSAT3," J. Opt. Soc. Korea 12, 262-268 (2008).

3. B. Brixner, "A 3048-mm, F/2.5, flat-field Schmidt telescope design,” Appl. Opt. 6, 1069-1072 (1967).

4. L. Epstein, "Improved geometry for the all reflecting Schmidt telescope," Appl. Opt. 12, 926-928 (1973).

5. J. H. Pan, The Design, Manufacture and Test of the Aspheric Optical Surfaces (Science Press, Beijing, China, 1994), pp. 157-167.

6. J. P. Bu, W. J. Tian, and X. J. Yang, "A novel design of off-axis three-mirror reflective optical system," Acta Photonica Sinica 35, 608-610 (2006). 